\title{
COMPLETE RIEMANNIAN MANIFOLD MINIMALLY IMMERSED IN A UNIT SPHERE $S^{n+p}(1)$
}

\author{
By \\ Qing-ming Cheng and Yuen-da WANG
}

\section{Innroduction.}

Let $M^{n}$ be an $n$-dimensional Riemannian manifold which is minimally immersed in a unit sphere $S^{n+p}(1)$ of dimension $n+p$. If $M^{n}$ is compact, then many authors studied them and obtained many beautiful results (for examples [1], [3], [4], [5] and [6]). In this paper, we make use of Yau's maximum principle to extend these results to complete manifolds with Ricci curvature bounded from below.

\section{Preliminaries.}

Let $M^{n}$ be an $n$-dimensional Riemannian manifold which is minimally immersed in a unit sphere $S^{n+p}(1)$ of dimension $n+p$. Then the second fundamental form $h$ of the immersion is given by $h(X, Y)=\tilde{\nabla}_{X} Y-\nabla_{X} Y$ and it satisfies $h(X, Y)=h(Y, X)$, where $\tilde{\nabla}$ and $\nabla$ denote the covariant differentiation on $S^{n+p}(1)$ and $M^{n}$ respectively, $X$ and $Y$ are vector fields on $M^{n}$. We choose a local field of orthonormal frames $e_{1}, \cdots, e_{n}, \cdots, e_{n+p}$ in $S^{n+p}(1)$ such that, restricted to $M^{n}$, the vector $e_{1}, \cdots, e_{n}$ are tangent to $M^{n}$. We use the following convention on the range of indices unless otherwise stated: $A, B, C, \cdots=1,2, \cdots$, $n+p ; i, j, k, \cdots=1,2, \cdots, n ; \alpha, \beta, \cdots=n+1, \cdots, n+p$. And we agree that repeated indices under a summation sign without indication are summed over the respective range. With respect to the frame field of $S^{n+p}(1)$ chosen above, let $\widetilde{\omega}_{1}, \cdots, \widetilde{\omega}_{n+p}$ be the dual frames. Then structure equations of $S^{n+p}(1)$ are given by

$$
\begin{gathered}
d \widetilde{\omega}_{A}=\sum \widetilde{\omega}_{A B} \wedge \widetilde{\omega}_{B}, \quad \widetilde{\omega}_{A B}+\widetilde{\omega}_{B A}=0, \\
d \widetilde{\omega}_{A B}=\sum \tilde{\omega}_{A C} \wedge \widetilde{\omega}_{C B}-\widetilde{\omega}_{A} \wedge \widetilde{\omega}_{B} .
\end{gathered}
$$

Restricting these forms to $M^{n}$, we have the structure equations of the immersion :

$$
\begin{gathered}
\omega_{a}=0, \\
\omega_{i \alpha}=\sum h_{i j}^{\alpha} \omega_{j}, \quad h_{i j}^{\alpha}=h_{j i}^{\alpha},
\end{gathered}
$$

$\overline{\text { Received November 2, }}$ 1987. Revised March 22, 1988 


$$
\begin{gathered}
d \omega_{i}=\sum \omega_{i j} \wedge \omega_{j}, \quad \omega_{i j}+\omega_{j i}=0, \\
d \omega_{i j}=\sum \omega_{i k} \wedge \omega_{k j}-\Omega_{i j}, \quad \Omega_{i j}=\frac{1}{2} \sum R_{i j k l} \omega_{k} \wedge \omega_{l}, \\
R_{i j k l}=\left(\delta_{i k} \delta_{j l}-\delta_{i l} \delta_{j k}\right)+\sum\left(h_{i k}^{\alpha} h_{j l}^{\alpha}-h_{i l}^{\alpha} h_{j k}^{\alpha}\right), \\
d \omega_{\alpha \beta}=\sum \omega_{\alpha \gamma} \wedge \omega_{\gamma \beta}-\Omega_{\alpha \beta}, \quad \Omega_{\alpha \beta}=\frac{1}{2} \sum R_{\alpha \beta i j} \omega_{i} \wedge \omega_{j}, \\
R_{\alpha \beta i j}=\sum\left(h_{i k}^{\alpha} h_{k j}^{\beta}-h_{j k}^{\alpha} h_{k i}^{\beta}\right) .
\end{gathered}
$$

Then, the second fundamental form $h$ can be written as

$$
h\left(e_{i}, e_{j}\right)=\Sigma h_{i j}^{\alpha} e_{\alpha} .
$$

If we define $h_{i j k}^{\alpha}$ by

$$
\sum h_{i j k}^{\alpha} \omega_{k}=d h_{i j}^{\alpha}+\sum h_{i k}^{\alpha} \omega_{k j}+\sum h_{k j}^{\alpha} \omega_{k i}+\sum h_{i j}^{\beta} \omega_{\beta \alpha},
$$

then, from (2.2), (2.3) and (2.4), we have $h_{i j k}^{\alpha}=h_{i k j}^{\alpha}$.

Let $K_{N}$ be the square of the length of curvature tensor of the normal bundle, that is,

$$
K_{N}=\sum\left(\sum_{k}\left(h_{i k}^{\alpha} h_{k j}^{\beta}-h_{j k}^{\alpha} h_{k i}^{\beta}\right)\right)^{2}
$$

Setting

$$
L_{N}=\Sigma\left(\sum_{i j} h_{i j}^{\alpha} h_{i j}^{\beta}\right)^{2} .
$$

In this paper, we used the notations in [2].

LEMMA 1 ([4] or [6]). If $M^{n}$ is an n-dimensional Riemannian manifold minimally immersed in a unit sphere $S^{n+p}(1)$ of dimension $n+p$. Then, Simons' equation

$$
\begin{aligned}
\frac{1}{2} \Delta\|h\|^{2} & =\Sigma\left(h_{i j k}^{\alpha}\right)^{2}+\Sigma\left(h_{i j}^{\alpha} h_{k l}^{\alpha} R_{l i j k}+h_{i j}^{\alpha} h_{i l}^{\alpha} R_{l k j k}\right)-\frac{1}{2} K_{N} \\
& =\Sigma\left(h_{j i k}^{\alpha}\right)^{2}-K_{N}-L_{N}+n\|h\|^{2}
\end{aligned}
$$

holds good and

$$
\|h\|^{2}=n(n-1)-R,
$$

where $\|h\|$ denotes the length of the second fundamental form $h$ such that $\|h\|^{2}=$ $\Sigma\left(h_{i j}^{\alpha}\right)^{2}$ and $R$ is the scalar curvature of $M^{n}$.

LEMMA 2 ([7]). Let $M^{n}$ be a complete Riemannian manifold with Ricci curvature bounded from below. Let $f$ be a $C^{2}$-function bounded from above on $M^{\prime n}$, then for all $\varepsilon>0$, there exists a point $x$ in $M^{n}$ at which 


$$
\sup f-\varepsilon<f(x)
$$

$\|\nabla f\|<\varepsilon$,

$$
\Delta f<\varepsilon .
$$

\section{Main results.}

THEOREM 1. Let $M^{n}$ be an n-dimensional complete Riemannian manifold minimally immersed in a unit sphere $S^{n+p}(1)$ of dimension $n+p$ with Ricci curvature bounded from below. Then either $M^{n}$ is totally geodesic, in this case, $M^{n}=S^{n}(1)$ holds locally or inf $R \leqq n(n-1)-n /(2-1 / p)$.

PROOF. According to Lemma 1, we have

$$
\frac{1}{2} \Delta\|h\|^{2}=\Sigma\left(h_{i j k}^{\alpha}\right)^{2}-K_{N}-L_{N}+n\|h\|^{2},
$$

Because

$$
\sum_{i j}\left(\sum_{k}\left(h_{i k}^{\alpha} h_{k j}^{\beta}-h_{j k}^{\alpha} h_{k i}^{\beta}\right)\right)^{2} \leqq 2 \sum_{i j}\left(h_{i j}^{\alpha}\right)^{2} \sum_{i j}\left(h_{i j}^{\beta}\right)^{2}
$$

we get

$$
\begin{aligned}
K_{N} & =\sum\left(\sum_{k}\left(h_{i k}^{\alpha} h_{k j}^{\beta}-h_{j k}^{\alpha} h_{k i}^{\beta}\right)\right)^{2} \leqq 2 \sum_{i j}\left(\sum_{i j}\left(h_{i j}^{\alpha}\right)^{2} \Sigma\left(h_{i j}^{\beta}\right)^{2}\right) \\
& =2\|h\|^{4}-2 \sum\left(\sum_{i j}\left(h_{i j}^{\alpha}\right)^{2}\right)^{2} .
\end{aligned}
$$

(3.1) and (3.3) imply

$$
\frac{1}{2} \Delta\|h\|^{2} \geqq\|h\|^{2}\left\{n-(2-1 / p)\|h\|^{2}\right\} . \quad(\text { see }[1] \text { or }[4])
$$

If $\inf R>n(n-1)-n /(2-1 / p)$, from Lemma 1, we have

$$
\|h\|^{2}=n(n-1)-R
$$

Hence, $\|h\|^{2}$ is bounded. We define $f=\|h\|^{2}, F=(f+a)^{1 / 2}$ (where $a>0$ is any positive constant number). $F$ is bounded because $\|h\|^{2}$ is bounded.

$$
\begin{gathered}
d F=\frac{1}{2}(f+a)^{-1 / 2} d f, \\
\Delta F=\frac{1}{2}\left\{-\frac{1}{2}(f+a)^{-3 / 2}\|d f\|^{2}+(f+a)^{-1 / 2} \Delta f\right\} \\
=\frac{1}{2}\left\{-2\|d F\|^{2}+\Delta f\right\}(f+a)^{-1 / 2} \\
=\frac{1}{2 F}\left\{-2\|d F\|^{2}+\Delta f\right\} .
\end{gathered}
$$


Hence, $F \Delta F=-\|d F\|^{2}+\frac{1}{2} \Delta f$, namely,

$$
\frac{1}{2} \Delta f=F \Delta F+\|d F\|^{2} .
$$

Applying the Lemma 2 to $F$, we have for all $\varepsilon>0$, there exists a point $x$ in $M^{n}$ such that at $x$

$$
\begin{gathered}
\|d F(x)\|<\varepsilon . \\
\Delta F(x)<\varepsilon, \\
F(x)>\sup F-\varepsilon .
\end{gathered}
$$

(3.6), (3.7) and (3.8) imply

$$
\frac{1}{2} \Delta f<\varepsilon^{2}+F \varepsilon=\varepsilon(\varepsilon+F) \quad(\text { from } F>0) .
$$

We take the sequence $\left\{\boldsymbol{\varepsilon}_{m}\right\}$ such that $\boldsymbol{\varepsilon}_{m} \rightarrow 0(m \rightarrow \infty)$ and for all $m$, there exists a point $x_{m}$ in $M^{n}$ such that (3.7), (3.8) and (3.9) hold good. Hence, $\boldsymbol{\varepsilon}_{m}\left(\boldsymbol{\varepsilon}_{m}+F\left(x_{m}\right)\right) \rightarrow 0(m \rightarrow \infty)$ (because $F$ is bounded).

On the other hand, from (3.9),

$$
F\left(x_{m}\right)>\sup F-\varepsilon_{m} .
$$

Because $F$ is bounded, $\left\{F\left(x_{m}\right)\right\}$ is a bounded sequence, we get

$$
F\left(x_{m}\right) \longrightarrow F_{0} \text { (if necessary, we can choose subsequence). }
$$

Hence,

$$
F_{0} \geqq \sup F
$$

According to the properties of supremum, we have

$$
F_{0}=\sup F \text {. }
$$

From the definition of $F$, we get

$$
f\left(x_{m}\right) \longrightarrow f_{0}=\sup f_{0} \quad\left(\text { from } F_{0}=\sup F\right)
$$

From (3.4) and (3.10), we obtain

$$
\begin{gathered}
f[n-(2-1 / p) f] \leqq \frac{1}{2} \Delta f<\varepsilon^{2}+\varepsilon F, \\
f\left(x_{m}\right)\left[n-(2-1 / p) f\left(x_{m}\right)\right]<\varepsilon_{m}^{2}+\varepsilon_{m} F\left(x_{m}\right) \leqq \varepsilon_{m}^{2}+\varepsilon_{m} F_{0},
\end{gathered}
$$

Let $m \rightarrow \infty$, we have $\varepsilon_{m} \rightarrow 0, f\left(x_{m}\right) \rightarrow f_{0}$. Hence,

$$
f_{0}\left[n-(2-1 / p) f_{0}\right] \leqq 0
$$


1) If $f_{0}=0$, we have $f=\|h\|^{2} \equiv 0$, hence $M^{n}$ is totally geodesic, from [4], we know $M^{n}=S^{n}(1)$ holds locally.

2) If $f_{0}>0$, we have

$$
\begin{gathered}
n-(2-1 / p) f_{0} \leqq 0, \\
f_{0} \geqq n /(2-1 / p),
\end{gathered}
$$

that is, $\sup \|h\|^{2} \geqq n /(2-1 / p)$. From $(2.15)$,

$$
\inf R \leqq n(n-1)-n /(2-1 / p)
$$

This completes the proof of Theorem 1 .

THEOREM 2. Let $M^{n}$ be an n-dimensional complete Riemannian manifold with Ricci curvature bounded from below which is minimally immersed in a unit sphere $S^{n+p}(1)$ of dimension $n+p$. If $K_{N}=0$, then, either $M^{n}$ is totally geodesic and $M^{n}=S^{n}(1)$ holds locally or inf $R \leqq n(n-2)$.

Proof. Because $K_{N}=0$ if and only if, for any $\alpha, \beta$,

$$
\begin{gathered}
\sum_{i j}\left(\sum_{k}\left(h_{i k}^{\alpha} h_{k j}^{\beta}-h_{j k}^{\alpha} h_{k i}^{\beta}\right)\right)^{2}=0, \\
\frac{1}{2} \Delta\|h\|^{2} \geqq-L_{N}+n\|h\|^{2} \\
\geqq n\|h\|^{2}-\|h\|^{4} \\
=\|h\|^{2}\left(n-\|h\|^{2}\right) \quad \text { (see [4]) }
\end{gathered}
$$

Hence, using the same arguments as Theorem 1, we have $\|h\|^{2}=0$ or sup $\|h\|^{2}$ $\geqq n$. Thus, from Lemma 1 , we know either $M^{n}$ is totally geodesic and $M^{n}=$ $S^{n}(1)$ holds locally or $\inf R \leqq n(n-2)$.

\section{References}

[1] Chern, S.S., Do Darmo, M. and Kobayashi, S., Minimal submanifold of a sphere with second fundamental form of constant length, Functional Analysis and Related Fields, Springer, 1970, 59-75.

[2] Itoh, T., On Veronese manifolds, J. Math. Soc. Japan, 27 (1975), 497-506.

[3] Kenmotsu, K., Tohoku Math. J. 22 (1970), 240-248.

[4] Kenmotsu, K., Tanno, S. and Ogiue, K., Surveys in Geometry 1981/82, Minimal Surfaces I, p. 0-33-48, In Japanese.

[5] Lawson, H.B., Local rigidity theorems for minimal hypersurfaces, Ann. of Math. 89 (1969), 187-197.

[6] Simons, J., Minimal varieties in Riemannian manifolds, Ann. of Math. 88 (1968), 62-105. 
[7] Yau, S.-T., Harmonic functions on complete Riemannian manifolds, Comm. Pure Appl. Math. 28 (1975), 201-228.

Deparment of Mathematics

Northeast University of Technology

Shenyang, Liaoning, China 